\title{
Traffic Model for Teleconsultation Based on the Service Characteristics
}

\author{
Xin Yang ${ }^{1,2}$, Ningbo Zhang ${ }^{1,2}$ and Guixia Kang ${ }^{1,2}$ \\ ${ }^{1}$ Key Laboratory of Universal Wireless Communications, Ministry of Education, Beijing University of Posts and \\ Telecommunications, Beijing 100876, China \\ ${ }^{2}$ Wuxi BUPT Sensory Technology and Industry Institute CO.LTD.
}

\begin{abstract}
The rapid development of numerous emerging network services poses a challenge to the network resources allocation with their bandwidth requirements and statistical characteristics. Network traffic modeling plays an important role in network resources allocation which can be used to guarantee Quality-of-Service (QoS) of these new services. In this study, we analyzed the service composition and traffic characteristics of the spreading teleconsultation service and establish a traffic model. The obtained experimental results show that the proposed methodology which combines discrete autoregressive model (DAR) and wavelet analysis provides good accuracy in teleconsultation service traffic modeling.
\end{abstract}

Keywords-service traffic model; video traffic; discrete autoregressive model; wavelet model

\section{INTRODUCTION}

Network traffic modeling plays an essential role in network design and simulation. Besides generating traffic for network performance evaluation, traffic models can be used to guarantee Quality-of-Service (QoS) of network services [1].

Different network services have different traffic characteristics and Quality-of-Service (QoS) requirements. Therefore, it is necessary to establish traffic models based on service characteristics for various network services. For example, the WWW traffic modeling pay more attention to client-based traces [2], whereas video traffic modeling mainly focuses on video format [3].

With the rapid development of Internet, many emerging network services have arised such as smart home, Virtual Reality and telemedicine. In this case, traffic models establishment should not be limited to traditional network services. It is necessary to establish traffic models for emerging network services. Numerous traffic models have been proposed in the past, but few studies have considered traffic model of these new network services. Therefore, the goal of this work is to develop a traffic model for the emerging teleconsultation service based on its service characteristics.

Teleconsultation is widely used in telemedicine which provides real-time video communication between medical staff and patient for delivery healthcare. Other health-related data transmission is operated at the same time, such as medical images and text transmission. Due to the full transmission of video during the teleconsultation, the video traffic takes a great proportion of the total traffic. Consequently, the vital task is to study the characteristics of the teleconsultation video and develop traffic model for it.

Some traffic models for video have been studied in previous researches. Most models are based on autoregressive models and their combinations, which fit the autocorrelation of video traffic well with low complexity [4]. I. Spanou proposed Discrete Autoregrassive modeling which is based on scene change detection [5]. Markov model with high complexity for 3D video is put forward in [6].

Teleconsultation video scenes has slight movement and unchanged background similar to video conference, so we try to use Discrete Autoregressive Model (DAR) proposed in [10]. However, the DAR(1) model can not capture the behavior of teleconsultation video well. Therefore we propose a method combines wavelet and $\operatorname{DAR}(1)$ to improve the probability density function accuracy of the teleconsultation video traffic model. The experimental results show that the proposed methodology outperforms DAR(1) model.

The rest of the paper is organized as follows. In Section II, we introduce the teleconsultation service and traffic model for teleconsultation video. The performance evaluation and comparision are presented in Section III. Finally, Section IV concludes this paper.

\section{Teleconsultation SerVice Traffic Model}

\section{A. Service Composition}

Telemedicine is an effective way to sharing health resources, it offers an efficient tool for health practitioners. Health practitioners share expertise during remote medical boards and diagnosis, especially for difficult cases [7]. Teleconsultation is widely used in telemedicine due to the convenience. According to prior studies, telemedicine or teleconsultation using videoconferencing can improve access and quality of clinical care available to rural patients [8]. Before establishing traffic model for teleconsultation, we first introduce the service composition of teleconsultation.

As Figure 1 shows, teleconsultation service provides video communication, medical images and teleconsultation text transmission between patient and doctor. Teleconsultation holds about ten to fifteen minutes. At the beginning of the teleconsultation process, medical images are transmitted and then there will be teleconsultation text transmission. Video transmission occurs throughout the process, so it is obvious that 


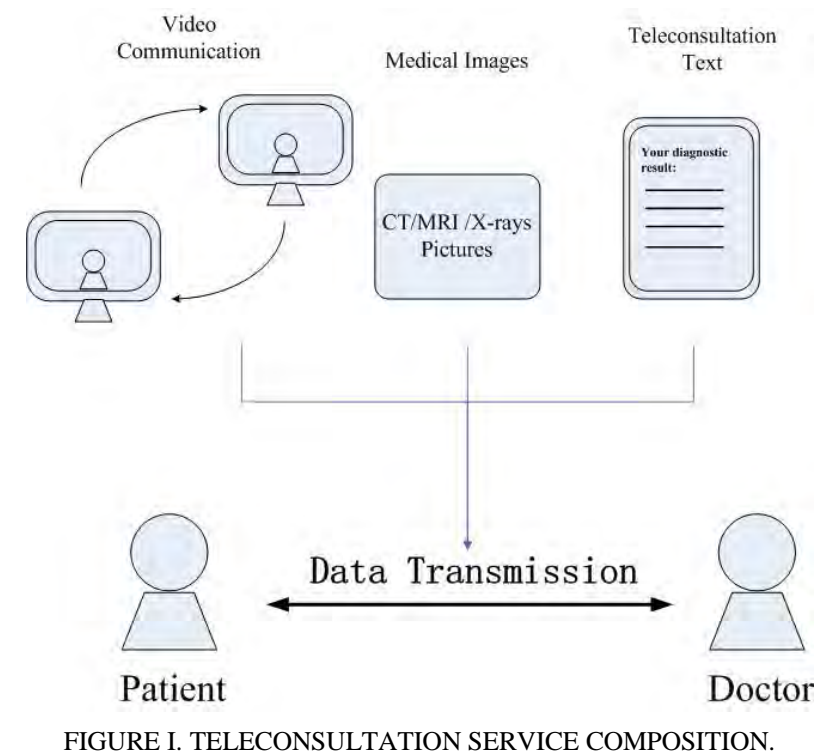

video traffic takes a great burden of the total traffic. Hence, developing traffic model for teleconsultation video plays an important role in service traffic model establishment.

Teleconsultation video consists of video scenes in which a person is talking to doctor with slight movement and almost unchanged background. We downloaded ten videos with five different people with slight movement from Internet, each video lasts about ten minutes to simulate the teleconsultation video and generate their corresponding H.264 AVC video traces with Elecard StreamEye Tools. Subsequent analysis of the model is based on the ten video traces.

H.264/AVC standard is the most widely used video coding standard for streaming videos, mobile/handheld applications, HDTV broadcasting [9]. In order to achieve a higher compression ratio, H.264/AVC standard divided video frames into three categories: I-frames, B-frames and P-frames.

A good traffic model is expected to capture the statistical characteristics of video sequences, such as frame size distribution and autocorrelation function (ACF) [1]. Since each frame type has different statistical characteristics, their traffic models will be developed separately. Teleconsultation video scenes has slight movement and unchanged background similar to video conference, so we try to use Discrete Autoregressive Model (DAR) proposed in [10].

\section{B. Discrete Autoregressive Process Model}

Discrete autoregressive model is proved to be accurate on fitting of the marginal distribution of video frame sizes of H.264 video traces [10].

A discrete autoregressive model of order $p$, denoted as $\mathrm{DAR}(\mathrm{p})$, generates a stationary sequence of discrete random variables with an arbitrary probability distribution and with an autocorrelation structure similar to that of an autoregressive model [11].

$\operatorname{DAR}(1)$ is a special case of a $\operatorname{DAR}(\mathrm{p})$ process and it is defined as the linear model:

$$
X_{n}=V_{n} X_{n-1}+\left(1-V_{n}\right) Y_{n}
$$

$\left\{X_{n}\right\}$ is a sequence of random variables. $\left\{V_{n}\right\}$ and $\left\{Y_{n}\right\}$ are two sequences of independent random variables. $\left\{V_{n}\right\}$ takes the values 0 and 1 with probability $1-\rho$ and $\rho$ respectively. $\left\{Y_{n}\right\}$ has a discrete state space $\mathrm{S}$ and $\mathrm{P}\left\{Y_{n}=\mathrm{i}\right\}=\pi(\mathrm{i})$. A $\operatorname{DAR}(1)$ process is a Markov chain with a discrete state space $\mathrm{S}$ and a transition matrix:

$$
P=\rho I+(1-\rho) Q
$$

$\rho$ is the lag -1 autocorrelation coefficient, I is the identity matrix and $\mathrm{Q}$ is a matrix with $\mathrm{Q}_{i j}=\pi(\mathrm{j})$ for $\mathrm{i}, \mathrm{j} \in \mathrm{S}$. The $\mathrm{Q}$ matrix consists of the negative binomial probabilities $\left\{f_{0}, f_{1}, \ldots f_{k}, F_{k}\right\}$, where $\mathrm{K}$ is the peak rate and $\mathrm{F}_{k}=\sum_{k>K} f_{k}$. Therefore, four parameters are needed for traffic model, the lag -1 autocorrelation coefficient, the peak rate , mean and variance of the offered traffic. The DAR(1) model can be used with any marginal distribution [10], we chose gamma distribution in our work.

Q-Q plot is a graph of set of pairs which is used for model evaluation, the closer to the line $\mathrm{x}=\mathrm{y}$, the better match between actual and model traffic. As Figure 5-7 show, the DAR(1) model captures the behavior of the B-frames and P-frames well, but can not produce a good fit for I-frames. It is caused by different compression method of different video frames. I-frames use intra-frame compression, whereas P-frames and B-frames use inter-frame compression. Hence, I-frames size changes more widely than B-frames and P-frames. DAR model is based on the correlation of video frames, the correlation will be weak when the change becomes strong, so DAR model can not produce a good fit for I-frames. In order to improve the accuracy of Iframes traffic model, the wavelet model will be introduced in next section.

\section{DAR Wavelet Model}

The DAR(1) model can not provide good fit for I-frames due to the widely change range of I-frames. DAR model is more shuitable for traffic which has less detail. Teleconsultation video has slight movement, but I frame size is possible to become small or large when movement occurs. The wavelet transform analyze the signal by multi-scale refinement operation, so it can focus on any details of the I frames.

Wavelet analysis is based on a high-pass wavelet function which generates the detailed coefficients of the original signal, and a low-pass scaling filter which produces the approximation coefficients of the original signal. The process of wavelet transform is shown in the Figure 2 The establishment of Iframes traffic model is based on the statistical analysis of the wavelet transform coefficients and the wavelet reconstruction.

Since Harr wavelet transform is a typical wavelet transform for its simplicity and good performance, it can be used for traffic model. Haar scaling and wavelet functions are,

$$
\varphi(t)= \begin{cases}1, & 0 \leq t<1 \\ 0, & \text { otherwise }\end{cases}
$$




$$
\emptyset(\mathrm{t})=\left\{\begin{array}{cc}
1, & 0 \leq t<1 / 2 \\
-1, & 1 / 2 \leq t<1 \\
0, & \text { otherwise }
\end{array}\right.
$$

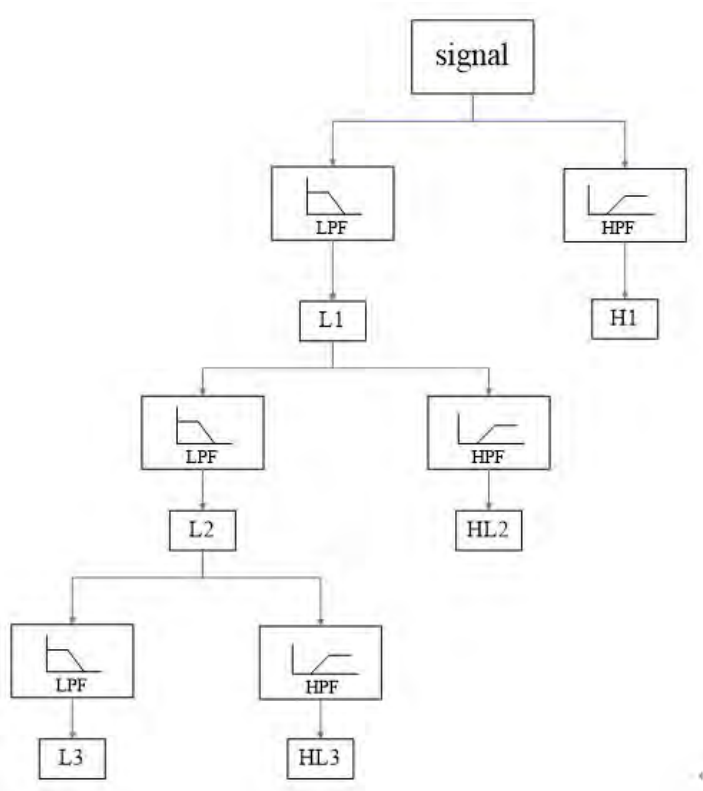

FIGURE II. PROCESS OF WAVELET TRANSFORM.

In order to develop a traffic model for I-frames, the analysis of the statistical properties of approximation and detailed coefficients is essential. For simplicity, we define approximation coefficients as $\mathrm{A}_{j}$ and detailed coefficients as $\mathrm{D}_{j}$ where $\mathrm{j}$ is the wavelet decomposition level. In Figure3 and Figure 4, we illustrate the distribution of one teleconsultation video we have downloaded as an example. Figure 3 shows little difference between probability density function of $A_{3}$ and gamma distribution. Experimental results show that Gamma distribution fits the actual histogram of $\mathrm{A}_{j}$ well.

As for $\mathrm{D}_{j}$, previous wavelet-based traffic modeling methods often model as zero-mean Gaussian variables [12]. However Laplacian distribution matches detailed coefficients well. Figure 4 displays the comparision of probability density function of $\mathrm{D}_{1}$ and Laplacian distribution. Laplace distribution has a wider range of values than Gaussian distribution, it is closer to the characteristics of actual $\mathrm{D}_{j}$.

\section{Simulation Results}

In this section, we provide the simulation results on teleconsultation video traffic model. We downloaded ten videos with five different people with slight movement from Internet, each video lasts about ten minutes to simulate the teleconsultation video. Corresponding H.264 AVC video traces are generated with Elecard StreamEye Tools. Video trace can show the size and type of each video frame.

Statistical tests were made with the use of Q-Q plots and Kullback-Leibler divergence tests [13]. The Q-Q plot is a powerful goodness-of-fit test, which can determine whether the data sets come from populations with another distribution. If traffic model provides good match for actual traffic, the points of the plot should fall approximately along a 45-degree reference line. The Kullback-Leibler divergence test is a measure of the difference between two probability distributions.

As explained in section II, DAR(1) with gamma marginal distribution can be used to develop traffic model for teleconsultation video. However Figure5-7 suggest that DAR(1) captures the behavior of the B-frames and P-frames well, but can not produce a good fit for I-frames. As Figure 5 shows, DAR model can not capture the characteristics well when $\mathrm{x}$ is less than 180 or greater than 250 . It is caused by the wide range of Iframes.

In order to improve the accuracy of I-frames traffic model, we proposed wavelet model. Taking complexity and accuracy into account, we set wavelet decomposition level to three. Based on $A_{3}$ which consistents with gamma distribution and $D_{1}, D_{2}$, $\mathrm{D}_{3}$ which consistents with Laplacian distribution, we can establish traffic model by using inverse wavelet transform.

For more accurate comparison between DAR(1) for I-frames and wavelet model for I-frames, we used ten teleconsultation videos to develop traffic model with Kullback-Leibler divergence(KLD) as test tool. The smaller the KLD value is, the more accurate the model is. In Table 1, we show the KLD of two models for ten teleconsultation videos. It is obvious that wavelet model performed better on establishing I-frames traffic model than DAR(1). It is because that wavelet transform retains more details than DAR model.

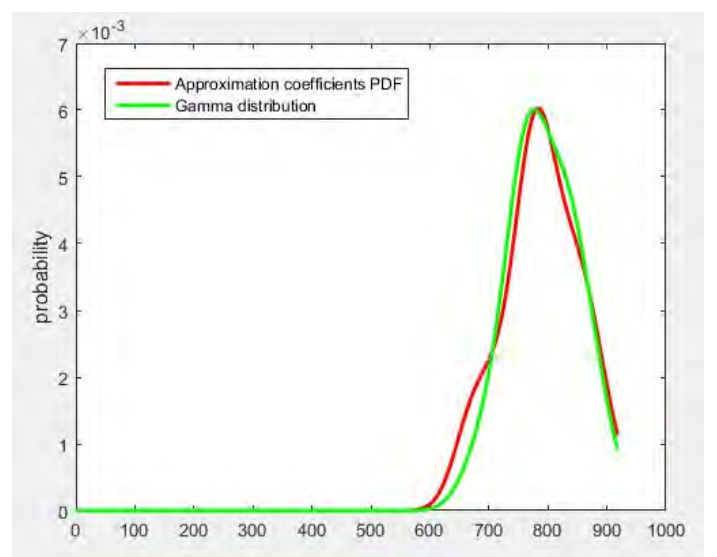

FIGURE III. COMPARISON BETWEEN PROBABILITY DENSITY FUNCTION OF APPROXIMATION COEFFICIENTS AND GAMMA DISTRIBUTION. 


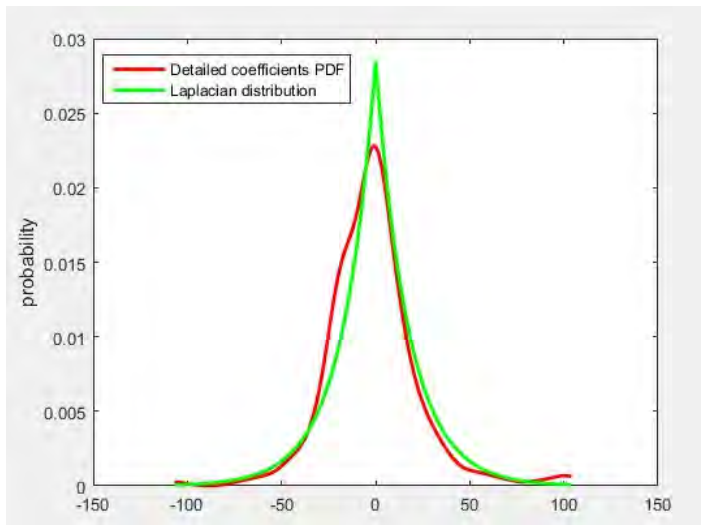

FIGURE IV. COMPARISON BETWEEN PROBABILITY DENSITY FUNCTION OF DETAILED COEFFICIENTS AND LAPLACIAN DISTRIBUTION.

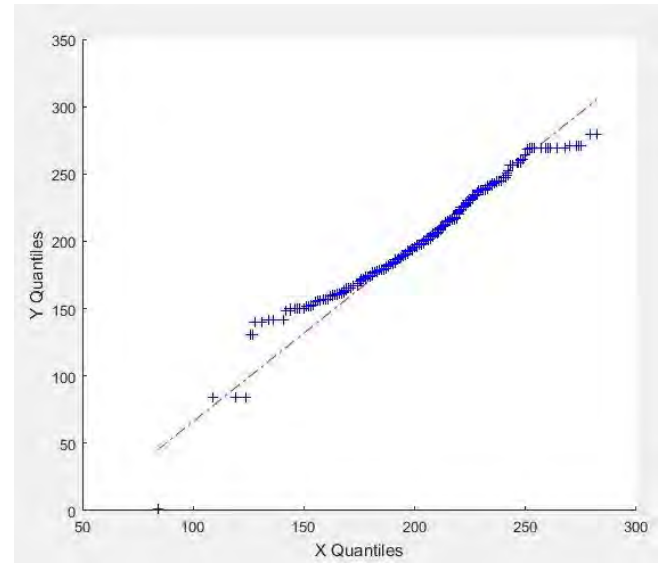

FIGURE V. Q-Q PLOT OF THE DAR(1) MODEL VERSUS THE ACTUAL VIDEO FOR THE I FRAMES.

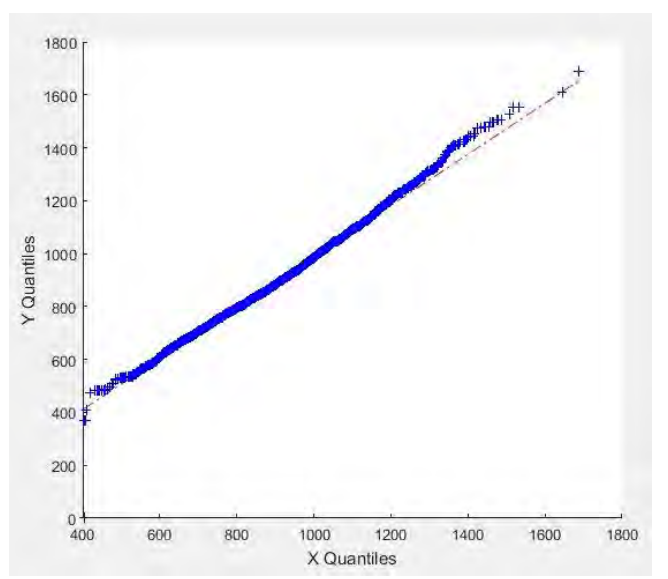

FIGURE VI. Q-Q PLOT OF THE DAR(1) MODEL VERSUS THE ACTUAL VIDEO FOR THE P FRAMES.

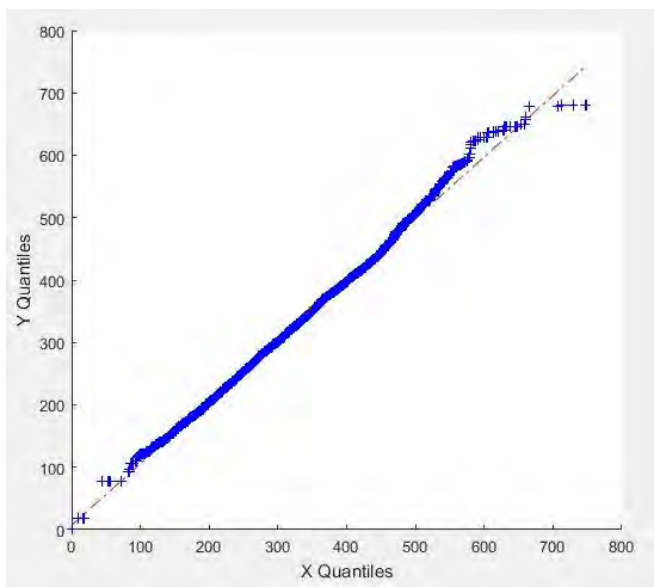

FIGURE VII. Q-Q PLOT OF THE DAR(1) MODEL VERSUS THE ACTUAL VIDEO FOR THE B FRAMES.

TABLE I. KULLBACK-LEIBLER DIVERGENCE OF DAR(1) AND WAVELET MODEL

\begin{tabular}{c|cc}
\hline $\begin{array}{c}\text { VIDEO } \\
\text { NUMBER }\end{array}$ & KLD OF DAR(1) & $\begin{array}{l}\text { KLD } \\
\text { MODEL }\end{array}$ \\
\hline 1 & 0.225 & 0.082 \\
2 & 0.102 & 0.056 \\
3 & 0.043 & 0.024 \\
4 & 0.041 & 0.032 \\
5 & 0.042 & 0.017 \\
6 & 0.073 & 0.045 \\
7 & 0.094 & 0.066 \\
8 & 0.042 & 0.022 \\
9 & 0.073 & 0.017 \\
10 & 0.122 & 0.067 \\
\hline
\end{tabular}

\section{CONCLUSIONS}

In this paper, the DAR wavelet (DARW) model is proposed to develop traffic model for emerging teleconsultation service. The DARW focus on teleconsultation video which has slight movement and unchanged background. Experimental results show that DARW performed better on establishing teleconsultation video traffic model than DAR. Future work should be oriented towards finding a distribution which can produce a better fit for wavelet coefficients.

\section{ACKNOWLEDGEMENT}

This work was supported by the Fundamental Research Funds for the Central Universities (2017RC06), the National Natural Science Foundation of China (61501056), and National Science and Technology Major Project of China (No. 2017ZX03001022).

\section{REFERENCES}

[1] M. Dai, Y. Zhang and D. Loguinov, "A Unified Traffic Model for MPEG4 and H.264 Video Traces," in IEEE Transactions on Multimedia, vol. 11, no. 5, pp. 1010-1023, Aug. 2009. 
[2] M. E. Crovella and A. Bestavros, "Self-similarity in World Wide Web traffic: evidence and possible causes," in IEEE/ACM Transactions on Networking, vol. 5, no. 6, pp. 835-846, Dec 1997.

[3] S. Tanwir and H. Perros, "A Survey of VBR Video Traffic Models," in IEEE Communications Surveys \\& Tutorials, vol. 15, no. 4, pp. 17781802, Fourth Quarter 2013.

[4] D. R. Marković, A. M. Gavrovska and I. S. Reljin, "4K video traffic analysis using seasonal autoregressive model for traffic prediction," 2016 24th Telecommunications Forum (TELFOR), Belgrade, 2016, pp. 1-4.

[5] I. Spanou, A. Lazaris and P. Koutsakis, "Scene change detection-based Discrete Autoregressive modeling for MPEG-4 video traffic," 2013 IEEE International Conference on Communications (ICC), Budapest, 2013, pp. 2386-239.

[6] L. Rossi, J. Chakareski, P. Frossard and S. Colonnese, "A Poisson Hidden Markov Model for Multiview Video Traffic," in IEEE/ACM Transactions on Networking, vol. 23, no. 2, pp. 547-558, April 2015.

[7] A. Chaabouni, J. Lambert, Y. Gaudeau, N. Tizon, D. Nicholson and J. M. Moureaux, "Quality assessment of MPEG-4 AVC/H.264 and HEVC compressed video in a telemedicine context," 2017 IEEE International Conference on Image Processing (ICIP), Beijing, 2017, pp. 3465-3469.

[8] T. Wang, J. Li, C. Zhu, Z. Hong, D. An, H. Yang et al., "Assessment of utilization and cost-effectiveness of telemedicine program in western regions of China: A 12-Year Study of 249 Hospitals Across 112 Cities", Te1emed e-Health., vol. 22, pp. 909-920, November 2016.

[9] C. Ram and S. Panwar, "Performance Comparison of High Efficiency Video Coding (HEVC) with H.264 AVC," 2017 13th International Conference on Signal-Image Technology \&\& Internet-Based Systems (SITIS), Jaipur, India, 2017, pp. 303-310.

[10] A. Lazaris and P. Koutsakis, "Modeling Video Traffic from Multiplexed H.264 Videoconference Streams," IEEE GLOBECOM 2008 - 2008 IEEE Global Telecommunications Conference, New Orleans, LO, 2008, pp. 16.

[11] P. A. Jacobs and P. A. W. Lewis, "Time Series Generated by Mixtures", Journal of Time Series Analysis, Vol. 4, No. 1, 1983,pp. 19-36.

[12] Sheng Ma and Chuanyi Ji, "Modeling heterogeneous network traffic in wavelet domain," in IEEE/ACM Transactions on Networking, vol. 9, no. 5, pp. 634-649, Oct 2001.

[13] K. P. Burnham and D. R. Anderson, "Model Selection and Multi-Model Inference ", Springer-Verlag, New York, 2002. 